\title{
Quality of Information Sources in Information Fusion
}

Frédéric Pichon, Didier Dubois and Thierry Denœux

\begin{abstract}
Pieces of information can only be evaluated if knowledge about the quality of the sources of information is available. Typically, this knowledge pertains to the source relevance. In this chapter, other facets of source quality are considered, leading to a general approach to information correction and fusion for belief functions. In particular, the case where sources may partially lack truthfulness is deeply investigated. As a result, Shafer's discounting operation and the unnormalised Dempster's rule, which deal only with source relevance, are considerably extended. Most notably, the unnormalised Dempster's rule is generalised to all Boolean connectives. The proposed approach also subsumes other important correction and fusion schemes, such as contextual discounting and Smets' $\alpha$-junctions. We also study the case where pieces of knowledge about the quality of the sources are independent. Finally, some means to obtain knowledge about source quality are reviewed.
\end{abstract}

\section{Introduction}

A central problem in various kinds of information systems is to determine the correct answer to a given question, for instance find the value of deterministic parameter $x$

Frédéric Pichon

Université d'Artois, EA 3926, Laboratoire de Génie Informatique et d'Automatique de l'Artois (LGI2A), F-62400 Béthune, France, e-mail: Frederic.Pichon@univ-artois.fr

Didier Dubois

Université Paul Sabatier, IRIT - Bureau 308, 118 Route de Narbonne, 31062 Toulouse Cedex 09, France, e-mail: Didier.Dubois@irit.fr

Thierry Denœux

Sorbonne Universités, Université de Technologie de Compiègne, CNRS, Heudiasyc (UMR 7253), BP20529, 60205 Compiègne Cedex, France, e-mail: Thierry.Denoeux@utc.fr

This chapter is a shorter and revised version of [24]. 
defined on a set $\mathcal{X}$ of possible values, from information provided by one or many sources.

Pieces of information about $x$ provided by several sources cannot be evaluated unless some meta-knowledge on the sources, i.e., knowledge about their quality, is available. Classically, such meta-knowledge relies on assumptions about the source relevance, where a relevant source is a source providing useful information about $x$. For instance, if the source is a sensor, then its response is typically relevant when it is in order and irrelevant when it is out of order. In particular, if a source $\mathfrak{s}$ is assumed to be relevant with probability $p$ and $\mathfrak{s}$ provides the testimony $x \in A$, then the information item $x \in A$ is considered not useful with probability $1-p$. This is know as the discounting of a piece of information [28, p. 251] [29] in the context of the theory of belief function [1, 28, 32] and the resulting state of knowledge is represented by a simple support function [28]: the weight $p$ is allocated to the fact of knowing only that $x \in A$ and the weight $1-p$ is allocated to knowing that $x \in \mathcal{X}$ (it is the probability of knowing nothing from the source). If two sources, with respective independent probabilities of relevance $p_{1}$ and $p_{2}$, both supply the information item $x \in A$, then one attaches reliability $p_{1}+p_{2}-p_{1} p_{2}$ to the statement $x \in A$, since one should deduce that $x \in A$ whenever at least one of the sources is relevant; this is the result obtained by Dempster's rule of combination $[1,28]$. This is actually the old problem of merging unreliable testimonies (see the entry "Probabilité" in [6]).

Beyond source relevance, it is proposed in this chapter to consider the case where knowledge on other facets of the quality of the sources is available. We start our study by adding the possibility to make assumptions about the truthfulness of the sources (Section 2). Then, we present a general framework able to deal with assumptions about various forms of source quality (Section 3 ). This study is conducted within the theory of belief functions and leads to a general approach to the fusion of belief functions. Related works, and especially practical means to apply our framework as well as relationships with some previous works, are reviewed in Section 4, before concluding in Section 5.

\section{Relevance and truthfulness}

The reliability of a source is usually assimilated to its relevance. In this section, we assume that reliability also involves another dimension: truthfulness. A truthful source is a source that actually supplies the information it possesses. A source may be non truthful in different ways. The crudest form of lack of truthfulness for a source is to declare the contrary of what it knows. It may also tell less or something different, even if consistent with its knowledge. For instance, a systematic bias of a sensor may be regarded as a form of lack of truthfulness. In this section, however, we shall only assume the crudest form of non truthfulness. 


\subsection{The case of a single source}

Consider the case where a single source $\mathfrak{s}$ provides information about $x$ and that this information is of the form $x \in A$, where $A$ is a proper non-empty ${ }^{1}$ subset of $\mathcal{X}$. If $\mathfrak{s}$ is assumed to be irrelevant, whatever may be its truthfulness, the information it provides is totally useless and can be replaced by the trivial information $x \in \mathcal{X}$. In contrast, if $\mathfrak{s}$ is assumed to be relevant and to tell the opposite of what it knows, then the actual information about $x$ can be retrieved: one should replace $x \in A$ by $x \in \bar{A}$, where $\bar{A}$ denotes the complement of $A$. Obviously, if $\mathfrak{s}$ is assumed to be relevant and truthful, then one infers that $x \in A$.

Formally, let $\mathcal{H}=\{(R, T),(R, \neg T),(\neg R, T),(\neg R, \neg T)\}$ denote the space of possible states of the source with respect to its relevance and truthfulness, where $R$ (resp. $T$ ) means that $\mathfrak{s}$ is relevant (resp. truthful). Then, following Dempster's approach [1], the above reasoning can be encoded by the multi-valued mapping $\Gamma_{A}: \mathcal{H} \rightarrow \mathcal{X}$ such that

$$
\begin{aligned}
\Gamma_{A}(R, T) & =A ; \\
\Gamma_{A}(R, \neg T) & =\bar{A} ; \\
\Gamma_{A}(\neg R, T) & =\Gamma(\neg R, \neg T)=\mathcal{X} .
\end{aligned}
$$

$\Gamma_{A}(h)$ interprets the testimony $x \in A$ in each state $h \in \mathcal{H}$ of $\mathfrak{s}$.

In general, the knowledge about the source relevance and truthfulness is uncertain. Specifically, each state $h \in \mathcal{H}$ may be assigned a subjective probability $\operatorname{prob}(h)$ such that $\sum_{h} \operatorname{prob}(h)=1$. In such case, the information item $x \in A$ yields the state of knowledge on $\mathcal{X}$ represented by a belief function in the sense of Shafer [28], with mass function $m^{\mathcal{X}}$ on $\mathcal{X}$ defined by

$$
\begin{aligned}
m^{\mathcal{X}}(A) & =\operatorname{prob}(R, T) \\
m^{\mathcal{X}}(\bar{A}) & =\operatorname{prob}(R, \neg T) ; \\
m^{\mathcal{X}}(\mathcal{X}) & =\operatorname{prob}(\neg R)=\operatorname{prob}(\neg R, T)+\operatorname{prob}(\neg R, \neg T) .
\end{aligned}
$$

A mass function $m^{\mathcal{X}}$ is formally a probability distribution on the power set of $\mathcal{X}$ (thus $\sum_{A \subseteq \mathcal{X}} m^{\mathcal{X}}(A)=1$ ). The quantity $m^{\mathcal{X}}(A)$ represents the weight allocated to the fact of knowing only that $x \in A$; it does not evaluate the likelihood of event $A$ like does the probability $\operatorname{prob}(A)$.

The testimony provided by the source may itself be uncertain. In particular, it may take the form of a mass function $m_{\mathfrak{s}}^{\mathcal{X}}$ on $\mathcal{X}$. Assume that $\mathfrak{s}$ is in a given state $h$, then each $m_{\mathfrak{s}}^{\mathcal{H}}(A)$ should be transferred to $\Gamma_{A}(h)$, yielding the mass function denoted $m^{\mathcal{X}}(B \mid h)$ and defined by

$$
m^{\mathcal{X}}(B \mid h)=\sum_{A: \Gamma_{A}(h)=B} m_{\mathfrak{s}}^{\mathcal{X}}(A), \quad \forall B \subseteq \mathcal{X} .
$$

\footnotetext{
${ }^{1}$ We consider as a source any entity that supplies a non-trivial and non-self-contradictory input.
} 
More generally, assume each state $h$ has a probability $\operatorname{prob}(h)$, then (7) implies that the state of knowledge on $\mathcal{X}$ is given by the following mass function

$$
m^{\mathcal{X}}(B)=\sum_{h} m^{\mathcal{X}}(B \mid h) \operatorname{prob}(h)=\sum_{h} \operatorname{prob}(h) \sum_{A: \Gamma_{A}(h)=B} m_{\mathfrak{s}}^{\mathcal{X}}(A) .
$$

Assuming that $p=\operatorname{prob}(R)$ and $q=\operatorname{prob}(T)$, and that the relevance of a source is independent of its truthfulness, leads then to transforming the mass function $m_{\mathfrak{S}}^{\mathcal{X}}$ into a mass function denoted by $m^{\mathcal{X}}$ and defined by:

$$
m^{\mathcal{X}}(A)=p q m_{\mathfrak{s}}^{\mathcal{X}}(A)+p(1-q) \bar{m}_{\mathfrak{s}}^{\mathcal{X}}(A)+(1-p) m_{\mathcal{X}}^{\mathcal{X}}(A), \quad \forall A \subseteq \mathcal{X}
$$

where $\bar{m}_{\mathfrak{s}}^{\mathcal{X}}$ is the negation of $m_{\mathfrak{s}}^{\mathcal{X}}$ [7], defined by $\bar{m}_{\mathfrak{S}}^{\mathcal{X}}(A)=m_{\mathfrak{S}}^{\mathcal{X}}(\bar{A}), \forall A \subseteq \mathcal{X}$, and $m_{\mathcal{X}}^{\mathcal{X}}$ the vacuous mass function defined by $m_{\mathcal{X}}^{\mathcal{X}}(\mathcal{X})=1$.

The discounting operation proposed by Shafer [28] to integrate the reliability of information sources is a special case of transformation (9), recovered for $q=1$ : it corresponds to a partially relevant source that is truthful. The negating operation [26] is also a special case recovered for $p=1$, which corresponds to a partially truthful source that is relevant. In particular, the negation of a mass function is obtained for $p=1$ and $q=0$ : it corresponds to a relevant source that is lying ${ }^{2}$.

Other forms of uncertain meta-knowledge about the source may be considered. In particular, it may be known only that the source state belongs to a subset $H$ of $\mathcal{H}$. This happens for instance if the source is assumed to be either relevant or truthful but not both, i.e., $H=\{(R, \neg T),(\neg R, T)\}$. In such case, one should deduce that $x \in \Gamma_{A}(H)$, where $\Gamma_{A}(H)$ denotes the image of $H$ under $\Gamma_{A}$ defined as

$$
\Gamma_{A}(H)=\bigcup_{h \in H}\left\{\Gamma_{A}(h)\right\} .
$$

Such non-elementary assumptions are actually not so interesting, since $\Gamma_{A}(H)=\mathcal{X}$ if $|H|>1$, where $|H|$ denotes the cardinality of $H$. Nonetheless, they are important in the case where multiple sources provide information items, which is the topic of the next section.

\subsection{The case of multiple sources}

Interpreting pieces of information provided by two sources requires making assumptions about their joint state with respect to their relevance and truthfulness. Let $\mathcal{H}_{i}$ denote the set of possible states of source $\mathfrak{s}_{i}, i=1,2$. The set of elementary joint state assumptions on sources is then $\mathcal{H}_{1: 2}=\mathcal{H}_{1} \times \mathcal{H}_{2}$ (we have $\left|\mathcal{H}_{1: 2}\right|=16$ ).

Let us first consider the simple case where each source $\mathfrak{s}_{i}$ provides a crisp piece of information $x \in A_{i}, i=1,2$, and where the two sources are assumed to be in some

2 The term "lying" is used as a synonym of "telling the negation of what is believed to be the truth", irrespective of the existence of any intention of a source to deceive. 
joint state $\mathbf{h}=\left(h^{1}, h^{2}\right) \in \mathcal{H}_{1: 2}$, with $h^{i} \in \mathcal{H}_{i}$ the state of $\mathfrak{s}_{i}, i=1,2$. The result of the merging of the information items $\mathbf{A}=\left(A_{1}, A_{2}\right) \subseteq \mathcal{X} \times \mathcal{X}$ provided by the sources depends on the assumption $\mathbf{h}$ made about their behaviour, and can be represented by a multi-valued mapping $\Gamma_{\mathbf{A}}: \mathcal{H}_{1: 2} \rightarrow \mathcal{X}$.

As one must conclude $x \in \Gamma_{A_{i}}\left(h^{i}\right)$ when $\mathfrak{s}_{i}$ tells $x \in A_{i}$ and is in state $h_{i} \in \mathcal{H}_{i}$, $i=1,2$, it is clear that one must deduce that $x \in \Gamma_{A_{1}}\left(h^{1}\right) \cap \Gamma_{A_{2}}\left(h^{2}\right)$ when the sources are assumed to be in the joint state $\mathbf{h}=\left(h^{1}, h^{2}\right) \in \mathcal{H}_{1: 2}$, i.e., we have

$$
\Gamma_{\mathbf{A}}(\mathbf{h})=\Gamma_{A_{1}}\left(h^{1}\right) \cap \Gamma_{A_{2}}\left(h^{2}\right) .
$$

Concretely, this means that

- if both sources are relevant, and

- they are both truthful, then one must conclude that $x \in A_{1} \cap A_{2}$;

- $\mathfrak{s}_{1}$ is truthful and $\mathfrak{s}_{2}$ is not, then one must conclude that $x \in A_{1} \cap \overline{A_{2}}$;

- $\mathfrak{s}_{2}$ is truthful and $\mathfrak{s}_{1}$ is not, then one must conclude that $x \in \overline{A_{1}} \cap A_{2}$;

- they are both non truthful, then one must conclude that $x \in \overline{A_{1}} \cap \overline{A_{2}}$;

- if source $\mathfrak{s}_{i}$ is relevant and the other source is irrelevant, then one must conclude that $x \in A_{i}$ (resp. $x \in \overline{A_{i}}$ ) if $\mathfrak{s}_{i}$ is truthful (resp. non truthful);

- if both sources are irrelevant, then one must conclude that $x \in \mathcal{X}$, irrespective of the elementary assumption made on their truthfulness.

Non-elementary assumptions $H \subseteq \mathcal{H}_{1: 2}$ can also be considered. Under such an assumption, one must conclude that $x \in \Gamma_{\mathbf{A}}(H)=\bigcup_{\mathbf{h} \in H}\left\{\Gamma_{\mathbf{A}}(\mathbf{h})\right\}$. Among the $2^{16}$ assumptions $H \subseteq \mathcal{H}_{1: 2}$, only a few are interesting (since, for instance, as soon as $H$ contains an elementary assumption such that both sources are non relevant, we have $\left.\Gamma_{\mathbf{A}}(H)=\mathcal{X}\right)$. In particular, by considering assumptions pertaining to the number of truthful and/or relevant sources, as well as to logical dependence between source states, it is possible to retrieve other Boolean binary connectives besides the four binary connectives $A_{1} \cap A_{2}, A_{1} \cap \overline{A_{2}}, \overline{A_{1}} \cap A_{2}$, and $\overline{A_{1}} \cap \overline{A_{2}}$ retrieved above.

Some interesting cases are as follows:

- Both sources are relevant, and at least one of them is truthful. This induces $x \in$ $A_{1} \cup A_{2}$. Note that this connective is also obtained by other assumptions, such as both sources are truthful, and exactly one of them is relevant.

- Both sources are relevant, exactly one of which is truthful, which yields $x \in A \triangle B$ (exclusive or).

- Both sources are relevant, and $\mathfrak{s}_{1}$ is truthful if and only if $\mathfrak{s}_{2}$ is so too. This results in $x \in(A \cap B) \cup(\bar{A} \cap \bar{B})$, which corresponds to the Boolean equivalence connective.

As a matter of fact, each logical connective can be retrieved from assumptions on the global quality of information sources, in terms of relevance and truthfulness. Accordingly, we will denote by $\otimes_{H}$ the set-theoretic connective associated to the assumption $H$.

Suppose now that assumption $H \subseteq \mathcal{H}_{1: 2}$ is made about the source quality and that $\mathfrak{s}_{i}$ supplies an uncertain testimony in the form of a mass function $m_{i}^{\mathcal{X}}, i=1,2$. 
Assume further that the sources are independent, where independence means the following: if we interpret $m_{i}^{\mathcal{X}}\left(A_{i}\right)$ as the probability that $\mathfrak{s}_{i}$ provides statement $x \in A_{i}$, then the probability that $\mathfrak{s}_{1}$ provides information item $x \in A_{1}$ and that $\mathfrak{s}_{2}$ provides conjointly information item $x \in A_{2}$ is $m_{1}^{\mathcal{X}}\left(A_{1}\right) \cdot m_{2}^{\mathcal{X}}\left(A_{2}\right)$. In such case, the state of knowledge on $\mathcal{X}$ is represented by the following mass function

$$
\begin{aligned}
m^{X}(B) & =\sum_{\mathbf{A}: \Gamma_{\mathbf{A}}(H)=B} m_{1}^{\mathcal{X}}\left(A_{1}\right) \cdot m_{2}^{\mathcal{X}}\left(A_{2}\right) \\
& =\sum_{\mathbf{A}: A_{1} \otimes_{H} A_{2}=B} m_{1}^{\mathcal{X}}\left(A_{1}\right) \cdot m_{2}^{\mathcal{X}}\left(A_{2}\right),
\end{aligned}
$$

which follows from the fact that if $\mathfrak{s}_{1}$ tells $x \in A_{1}$ and $\mathfrak{s}_{2}$ tells $x \in A_{2}$, then it is known under assumption $H$ that $x \in A_{1} \otimes_{H} A_{2}$.

Combination rule (11) encompasses the conjunctive rule [31] (the unnormalised version of Dempster's rule) and the disjunctive rule [7]. The former is retrieved by assuming that both sources are relevant and truthful, and the latter by assuming that, e.g., both sources are relevant and at least one of them is truthful. Note also that if $A_{1} \otimes_{H} A_{2}=\emptyset$ for two sets $A_{1}$ and $A_{2}$ such that $m_{1}^{\mathcal{X}}\left(A_{1}\right)>0$ and $m_{2}^{\mathcal{X}}\left(A_{2}\right)>0$, then this inconsistency pertains to a disagreement between the testimonies provided by the sources and the assumption $H$. In such case, a solution consists in rejecting $H$ and preferring an assumption compatible with the information provided by the sources. This will be discussed further in Section 4.1.

Assume now that the sources supply crisp testimonies of the form $x \in A_{1}$ and $x \in A_{2}$, but that the meta-knowledge regarding source quality is uncertain. Due to the interest of non-elementary assumptions, it seems useful to represent this uncertainty by a mass function, rather than a probability distribution, on $\mathcal{H}_{1: 2}$. The merging of $A_{1}$ and $A_{2}$ under $m^{\mathcal{H}_{1: 2}}$ result in a mass function on $\mathcal{X}$ defined by

$$
m^{\mathcal{X}}(B)=\sum_{H: A_{1} \otimes_{H} A_{2}=B} m^{\mathcal{H}_{1: 2}}(H)
$$

Remark that $m^{\mathcal{H}_{1: 2}}$ induces a probability distribution over the Boolean binary connectives attached to assumptions $H$ :

$$
p^{\mathcal{H}_{1: 2}}(\otimes)=\sum_{H: \otimes_{H}=\otimes} m^{\mathcal{H}_{1: 2}}(H) .
$$

In addition, let us stress that $m^{\mathcal{H}_{1: 2}}$ may carry a form of independence, which we call meta-independence between sources, different from the independence between sources defined above. Indeed, information supplied by the sources may be independent from each other, but pieces of meta-knowledge about the source state may not be independent. Formally, meta-independence between sources may be modelled by assuming that $m^{\mathcal{H}_{1: 2}}(H)=m^{\mathcal{H}_{1}}\left(H_{1}\right) m^{\mathcal{H}_{2}}\left(H_{2}\right)$ if $H=H_{1} \times H_{2}$ and $m^{\mathcal{H}_{1: 2}}(H)=0$ otherwise, which corresponds to evidential independence [28] between frames $\mathcal{H}_{1}$ and $\mathcal{H}_{2}$ with respect to $m^{\mathcal{H}_{1: 2}}$. 
A more general case, extending the combination operations (11) and (12), is obtained when both the testimonies supplied by independent sources and the metaknowledge about their quality are uncertain and represented by mass functions. This case induces the following mass function on $\mathcal{X}$ :

$$
\begin{aligned}
& m^{\mathcal{X}}(B)=\sum_{H} m^{\mathcal{H}_{1: 2}(H)} \sum_{\mathbf{A}: A_{1} \otimes_{H} A_{2}=B} m_{1}^{\mathcal{X}}\left(A_{1}\right) m_{2}^{\mathcal{X}}\left(A_{2}\right) \\
& =\sum_{\otimes} p^{\mathcal{H}_{1}: 2}(\otimes) \sum_{\mathbf{A}: A_{1} \otimes A_{2}=B} m_{1}^{\mathcal{X}}\left(A_{1}\right) m_{2}^{\mathcal{X}}\left(A_{2}\right) .
\end{aligned}
$$

This approach can be formally extended to the case of dependent sources, using the setting of [5]. It can also be readily extended to the case of $n>2$ sources that are partially truthful and relevant (see [23]). In theory, this latter extension may pose a computational issue (the belief function expressing meta-knowledge on the sources has a $2^{4^{n}}$ complexity in the general case). However, in practice, it can remain manageable as illustrated in [23], where special cases of the general combination rule (14) are considered. Besides the conjunctive and disjunctive rules, there are indeed other important combination schemes subsumed by (14). In particular, it is the case for the method, used in various approaches (see, e.g., [15, 27, 34, 2]), that consists in discounting sources and then combining them by the conjunctive rule, as shown in [24] and further discussed in [23]. The weighted average of belief functions and the combination rule corresponding to the assumption that $r$-out-of- $n$ sources are relevant, with $1 \leq r \leq n$, are also included in (14), as shown in [24] and [23], respectively.

\section{A general model of meta-knowledge}

In the preceding section, meta-knowledge concerned the relevance and the crudest form of lack of truthfulness of sources of information. However, in some applications, the lack of truthfulness may take a more refined form. Moreover, knowledge about the source quality may even be different from knowing their relevance and truthfulness. An approach to account for general source behaviour assumptions is thus necessary. Such an approach is proposed in this section.

\subsection{The case of a single source}

Suppose a source $\mathfrak{s}$ provides information about a parameter $y$ defined on a set $\mathcal{Y}$ of possible values and that this piece of information is of the form $y \in A$, for some $A \subseteq \mathcal{Y}$. Assume further that $\mathfrak{s}$ may be in one of $N$ elementary states instead of four as is the case in Section 2.1, i.e., we generalise the state space from $\mathcal{H}=\{(R, T),(R, \neg T),(\neg R, T),(\neg R, \neg T)\}$ to $\mathcal{H}=\left\{h_{1}, \ldots, h_{N}\right\}$. Moreover, we are 
interested by the value taken by a related parameter $x$ defined on a domain $\mathcal{X}$ and we have at our disposal some meta-knowledge that relate information item $y \in A$ supplied by $\mathfrak{s}$ to an information of the form $x \in B$, for some $B \subseteq X$, for each possible state $h \in \mathcal{H}$ of $\mathfrak{s}$. Namely, for each $A \subseteq \mathcal{Y}$, there is a multi-valued mapping $\Gamma_{A}: \mathcal{H} \rightarrow \mathcal{X}$ prescribing, for each elementary assumption $h \in \mathcal{H}$, how to interpret on $\mathcal{X}$ information item $y \in A$ provided by $\mathfrak{s}$. We also add the natural requirement that there exists $h \in \mathcal{H}$ such that $\Gamma_{A_{1}}(h) \neq \Gamma_{A_{2}}(h)$ for any two distinct subsets $A_{1}$ and $A_{2}$ of $\mathcal{Y}$. Incomplete assumptions $H \subseteq \mathcal{H}$ may also be considered, in which case information item $y \in A$ is interpreted as $x \in \Gamma_{A}(H)=\cup_{h \in H} \Gamma_{A}(h)$.

The setting of Section 2.1 is obtained as a particular case of this approach, by choosing $N=4, y=x$ and, e.g., $h_{1}=(R, T), h_{2}=(R, \neg T), h_{3}=(\neg R, T), h_{4}=$ $(\neg R, \neg T)$, in which case we have, for all $A \subseteq \mathcal{X}$ :

$$
\begin{aligned}
& \Gamma_{A}\left(h_{1}\right)=A, \\
& \Gamma_{A}\left(h_{2}\right)=\bar{A}, \\
& \Gamma_{A}\left(h_{3}\right)=\Gamma_{A}\left(h_{4}\right)=X .
\end{aligned}
$$

Example 1 provides another illustration of this approach.

Example 1 (Case $Y \neq X$, inspired from Janez and Appriou [13]). We are interested in finding the type $x$ of a given road, with $x$ taking its value in the set $X=\{$ track, lane, highway $\}$. A source $\mathfrak{s}$ provides information on this type, but it has a limited perception of the possible types of road and in particular is not aware of the existence of the type "lane", so that it provides information on the space $Y=\{$ track, highway $\}$. In addition, we know that this source discriminates between roads either using their width or their texture. If the source uses the road width, then when it says "track", we may only safely infer that the type is "track or lane" since tracks and lanes have similar width, and when it says "highway", we may infer "highway". On the other hand, if the source uses the road texture, then when it says "track", we may infer "track", and when it says "highway", we may only infer "highway or lane" since highways and lanes have similar textures.

This problem may be formalised using multi-valued mappings $\Gamma_{\text {track }}, \Gamma_{\text {highway }}$, and $\Gamma_{Y}$ from $\mathcal{H}=\{$ width, texture $\}$ to $\mathcal{X}$ defined as

$$
\begin{aligned}
\Gamma_{\text {track }}(\text { width }) & =\{\text { track }, \text { lane }\}, \\
\Gamma_{\text {track }}(\text { texture }) & =\{\text { track }\} \\
\Gamma_{\text {highway }}(\text { width }) & =\{\text { highway }\} \\
\Gamma_{\text {highway }}(\text { texture }) & =\{\text { lane }, \text { highway }\} \\
\Gamma_{Y}(\text { width }) & =X, \\
\Gamma_{Y}(\text { texture }) & =X .
\end{aligned}
$$

The proposed approach also makes it possible to model refined forms of lack of truthfulness, as explained hereafter. 
By taking a closer look at the non truthful state $\neg T$ considered in Section 2.1, we can remark that it corresponds to assuming that source $\mathfrak{s}$ tells the contrary of what it knows, whatever it is telling concerning each of the possible values $x_{i} \in \mathcal{X}$ that admits parameter $x$, since one must invert what $\mathfrak{s}$ tells for each of these values. For instance, let $\mathcal{X}=\left\{x_{1}, x_{2}, x_{3}, x_{4}\right\}$ and suppose that $\mathfrak{s}$ asserts $x \in A=\left\{x_{1}, x_{3}\right\}$, i.e., it tells that $x_{1}$ and $x_{3}$ are possible values for $x$ and that $x_{2}$ and $x_{4}$ are not possible values for $x$. Then, if $\mathfrak{s}$ is assumed to be in state $\neg T$, one must deduce that $x \in \bar{A}=\left\{x_{2}, x_{4}\right\}$, i.e., $x_{1}$ and $x_{3}$ are not possible values for $x$ and $x_{2}$ and $x_{4}$ are possible values for $x$.

Accordingly, we may introduce the notion of the truthfulness of a source for a value $x_{i} \in \mathcal{X}$ : a truthful (resp. non truthful) source for a value $x_{i} \in \mathcal{X}$ is a source that tells what it knows (resp. the contrary of what it knows) for this value. Hence, state $\neg T$ corresponds to the assumption that a source is non truthful for all values $x_{i} \in \mathcal{X}$. It is therefore a quite strong model of the lack of truthfulness of a source.

It seems interesting to consider more subtle forms of lack of truthfulness and in particular the assumption that a source $\mathfrak{s}$ could be non truthful only for some values $x_{i} \in \mathcal{X}$ (and truthful for all other values $x_{i} \in \mathcal{X}$ ), i.e., a kind of contextual lack of truthfulness. Let $B \subseteq \mathcal{X}$ be the set of values for which $\mathfrak{s}$ is truthful, and $\bar{B}$ the set of values for which it is not truthful. We will denote this state by $\ell_{B}$ (the state $\neg T$ corresponds then to the state $\ell_{\emptyset}$, and $T$ corresponds to $\ell_{\mathcal{X}}$ ). As shown in [26, Proposition 1], if $\mathfrak{s}$ asserts $x \in A$ for some $A \subseteq \mathcal{X}$, and is assumed to be in state $\ell_{B}$, for some $B \subseteq \mathcal{X}$, then one must deduce that $x \in(A \cap B) \cup(\bar{A} \cap \bar{B})$. We refer the reader to [26] for examples where such states $\ell_{B}$ may be relevant.

Considering the space of possible states $\ell_{B}$, i.e., $\mathcal{H}=\left\{\ell_{B} \mid B \subseteq \mathcal{X}\right\}$, and a testimony $x \in A$ supplied by a source, the above transformation can then be encoded by the multi-valued mapping $\Gamma_{A}: \mathcal{H} \rightarrow \mathcal{X}$ defined as:

$$
\Gamma_{A}\left(\ell_{B}\right)=(A \cap B) \cup(\bar{A} \cap \bar{B}), \quad \forall B \subseteq \mathcal{X} .
$$

A further refined model of lack of truthfulness can be obtained by being even more specific about the assumptions underlying the state $\neg T$, using the notions of positive and negative clauses [12, Chapter 8] told by the source. For instance, when $\mathfrak{s}$ declares that $x_{1}$ is a possible value for $x$, this is a positive clause told by the source, and when $\mathfrak{s}$ declares that $x_{2}$ is not a possible value for $x$, it is a negative clause. Accordingly, we may characterise the truthfulness of a source for each $x_{i} \in \mathcal{X}$, with respect to the polarity of the clauses it tells. Specifically, a source is said to be positively truthful (resp. non truthful) for a value $x_{i} \in \mathcal{X}$, when it declares that $x_{i}$ is a possible value for $x$ and knows that it is (resp. it is not). Hence, when a source is assumed to be positively truthful (resp. non truthful) for $x_{i} \in \mathcal{X}$ and declares that $x_{i}$ is a possible value for $x$, then one must deduce that it is (resp. it is not). Similarly, a source is said to be negatively truthful (resp. non truthful) for a value $x_{i} \in \mathcal{X}$, when it declares that $x_{i}$ is a not possible value for $x$ and knows that it is not (resp. it is). Hence, when a source is assumed to be negatively truthful (resp. non truthful) for $x_{i} \in \mathcal{X}$ and declares that $x_{i}$ is not a possible value for $x$, then one must deduce that it is not (resp. it is). Accordingly, state $\neg T$ corresponds to assuming that a source is positively and negatively non truthful for all values $x_{i} \in \mathcal{X}$. In that case, 
we make two strong assumptions: the context (set of values) concerned by the lack of truthfulness is the entire frame, and both polarities are concerned by the lack of truthfulness.

This suggests again to consider states corresponding to weaker assumptions on the lack of truthfulness. Two states are particularly interesting, as shown in [26]. The first one, denoted $p_{B}$, corresponds to the assumption that a source is (positively and negatively) truthful for all $x_{i} \in B$, and positively non truthful and negatively truthful for all $x_{i} \in \bar{B}$. Under such an assumption $p_{B}$, a testimony $x \in A$ is transformed into knowing that $x \in A \cap B$ [26, Proposition 2]. The second one, denoted $n_{B}$, corresponds to the assumption that a source is positively truthful and negatively non truthful for all $x_{i} \in B$, and (positively and negatively) truthful for all $x_{i} \in \bar{B}$. A testimony $x \in A$ is transformed into $x \in A \cup B$ under this latter assumption [26, Proposition 3].

These states also fit our approach since, e.g., the transformations associated to states $n_{B}$ can be represented by a multi-valued mapping $\Gamma_{A}$ from $\mathcal{H}=\left\{n_{B} \mid B \subseteq \mathcal{X}\right\}$ to $\mathcal{X}$ such that $\Gamma_{A}\left(n_{B}\right)=A \cup B$, for all $B \subseteq \mathcal{X}$. Let us remark that states $\ell_{B}, p_{B}$, and $n_{B}$, with associated transformations $(A \cap B) \cup(\bar{A} \cap \bar{B})$ (logical equality), $A \cap B$ (conjunction), and $A \cup B$ (disjunction), given testimony $x \in A$, are particular cases of a more general model of truthfulness assumptions yielding all possible binary Boolean connectives between testimony $A$ and context $B$, as detailed in [26].

The proposed approach can be further generalised to the case where both the information provided by the source and the meta-knowledge on the source are uncertain and represented by mass functions $m_{\mathfrak{s}}^{\mathcal{Y}}$ and $m^{\mathcal{H}}$, respectively. Since each mass $m_{\mathfrak{S}}^{\mathcal{Y}}(A)$ should be transferred to $\Gamma_{A}(H)$ under some hypothesis $H \subseteq \mathcal{H}$, the state of knowledge given $m_{\mathfrak{s}}^{\mathcal{Y}}$ and $m^{\mathcal{H}}$ is represented by a mass function defined by

$$
m^{\mathcal{X}}(B)=\sum_{H} m^{\mathcal{H}}(H) \sum_{A: \Gamma_{A}(H)=B} m_{\mathfrak{s}}^{\mathcal{Y}}(A), \quad \forall B \subseteq X,
$$

which generalises (8). Transformation (17) is referred to as Behaviour-Based Correction (BBC) as it modifies, or corrects [18], the information supplied by the source given our knowledge on its behaviour.

As detailed in [24], the BBC procedure generalises a deconditioning method known as the method by association of highest compatible hypotheses [14], which itself generalises a familiar operation of Dempster-Shafer theory, called conditional embedding (or ballooning extension) [29].

As shown recently in [26], the $\mathrm{BBC}$ procedure applied with states $n_{B}$ and $p_{B}$, respectively, can also be used to provide an interpretation to the contextual discounting and contextual reinforcement operations, which are two correction mechanisms introduced formally in [19]. In addition, when applied with state $\ell_{B}$, it can be used to obtain a contextual version of the negating operation [26]. 


\subsection{The case of multiple sources}

Consider now that two sources $\mathfrak{s}_{1}$ and $\mathfrak{s}_{2}$ provide information about $y$ and that each source may be in one of $N$ elementary states (those $N$ states are the same for both sources), with $\mathcal{H}_{i}$ the set of possible states of source $\mathfrak{s}_{i}, i=1,2$. Let $\mathcal{H}_{1: 2}=\mathcal{H}_{1} \times \mathcal{H}_{2}$ denote the set of elementary joint state assumptions on the sources.

Assume that each source $\mathfrak{s}_{i}$ provides a crisp piece of information $x \in A_{i}, i=1,2$. For the same reason as in Section 2.2, if the sources are assumed to be in state $\mathbf{h}=\left(h^{1}, h^{2}\right) \in \mathcal{H}_{1: 2}$, then we must conclude that $x \in \Gamma_{A_{1}}\left(h^{1}\right) \cap \Gamma_{A_{2}}\left(h^{2}\right)$, where $\Gamma_{A_{i}}$, $i=1,2$, are the mappings defined in Section 3.1. We can then define a multi-valued mapping $\Gamma_{\mathbf{A}}: \mathcal{H}_{1: 2} \rightarrow \mathcal{X}$, which assigns to each elementary hypothesis $\mathbf{h} \in \mathcal{H}_{1: 2}$ the result of the fusion of the information items $\mathbf{A}=\left(A_{1}, A_{2}\right) \subseteq \mathcal{X} \times \mathcal{X}$, as follows:

$$
\Gamma_{\mathbf{A}}(\mathbf{h})=\Gamma_{A_{1}}\left(h^{1}\right) \cap \Gamma_{A_{2}}\left(h^{2}\right) .
$$

As in Section 2.2, incomplete assumptions $H \subseteq \mathcal{H}_{1: 2}$ can be considered. In such case, one must conclude that $x \in \Gamma_{\mathbf{A}}(H)=\bigcup_{\mathbf{h} \in H}\left\{\Gamma_{\mathbf{A}}(\mathbf{h})\right\}$. More generally, suppose that the information supplied by the sources $\mathfrak{s}_{1}$ and $\mathfrak{s}_{2}$ and the meta-knowledge about their behaviour are uncertain and represented by mass functions $m_{1}^{\mathcal{Y}}, m_{2}^{\mathcal{Y}}$ and $m^{\mathcal{H}_{1: 2}}$, respectively. If furthermore the sources are assumed to be independent, then the fusion of $m_{1}^{\mathcal{Y}}$ and $m_{2}^{\mathcal{Y}}$ given $m^{\mathcal{H}_{1: 2}}$ result in the mass function on $\mathcal{X}$ defined by

$$
m^{\mathcal{X}}(B)=\sum_{H} m^{\mathcal{H}_{1: 2}}(H) \sum_{\mathbf{A}: \Gamma_{\mathbf{A}}(H)=B} m_{1}^{\mathcal{Y}}\left(A_{1}\right) m_{2}^{\mathcal{Y}}\left(A_{2}\right),
$$

which generalises (14). The combination (18) will be referred to as the BehaviourBased Fusion (BBF) rule.

Remark that this rule can be straightforwardly extended to the case of $n>2$ sources [23]. In addition, it can be extended to the case where sources $\mathfrak{s}_{i}, i=1, \ldots, n$ provide information on different spaces $\mathcal{Y}_{i}$ and have a different number $N_{i}$ of elementary states, which may be faced in some problems.

In [30], Smets introduced a family of combination rules representing the set of associative, commutative and linear operators for belief functions with the vacuous mass function as neutral element. This family, called the $\alpha$-conjunctions, depends on a parameter $\alpha \in[0,1]$. Operators in this family range between two rules based on Boolean operators as $\alpha$ decreases: the conjunctive rule (for $\alpha=1$ ), and the socalled equivalence rule [22] (for $\alpha=0$ ) based on the logical equivalence operator. Smets did not provide any clear interpretation for these rules for the cases $\alpha \in(0,1)$. As shown in [22], they are a particular case of the BBF rule: they correspond to assuming that either both sources tell the truth or they commit the same contextual lie $\ell_{B}$, with some particular weight depending on $\alpha$ and $B$. 


\subsection{The case of meta-independent sources}

Interestingly, the $\mathrm{BBC}$ procedure and the $\mathrm{BBF}$ rule can be recovered by defining particular valuation networks [16], representing the available pieces of information, and by propagating uncertainty in these networks, as stated by Lemmas 1 and 2 with associated valuation networks shown in Figures $1 \mathrm{a}$ and 1b, respectively.

Lemma 1 (BBC). Let $m^{\mathcal{H}}$ and $m_{\mathfrak{s}}^{\mathcal{Y}}$ be the mass functions and $\Gamma_{A}, A \subseteq \mathcal{Y}$, the mappings in (17). Let $\mathcal{Z}=2^{\mathcal{Y}}$ and let $z_{A}$ denote the element of $\mathcal{Z}$ corresponding to the subset $A$ of $\mathcal{Y}, \forall A \subseteq \mathcal{Y}$. For each $A \subseteq \mathcal{Y}$, let $\Gamma_{z_{A}}$ be the multi-valued mapping from $\mathcal{H}$ to $\mathcal{X}$ such that $\Gamma_{z_{A}}(h)=\Gamma_{A}(h)$ for all $h \in \mathcal{H}$. Let $m_{\mathfrak{S}}^{\mathcal{Z}}$ be the mass function defined by $m_{\mathfrak{s}}^{\mathcal{Z}}\left(\left\{z_{A}\right\}\right)=m_{\mathfrak{s}}^{\mathcal{Y}}(A)$, for all $A \subseteq \mathcal{Y}$. Let $m_{\Gamma}^{\mathcal{H} \times \mathcal{Z} \times \mathcal{X}}$ be the mass function defined by $m_{\Gamma}^{\mathcal{H}} \times \mathcal{Z} \times \mathcal{X}\left[\bigcup_{h \in \mathcal{H}, z_{A} \in \mathcal{Z}}\left(\{h\} \times z_{A} \times \Gamma_{z_{A}}(h)\right)\right]=1$.

We have, for all $B \subseteq \mathcal{X}$,

$$
\left(m_{\mathfrak{s}}^{\mathcal{Z}} @ m_{\Gamma}^{\mathcal{H} \times \mathcal{Z} \times \mathcal{X}} @ m^{\mathcal{H}}\right)^{\downarrow X}(B)=m^{\mathcal{X}}(B),
$$

where $m^{\mathcal{X}}$ is the mass function defined by (17) and where $@$ and $\downarrow$ denote, respectively, the unnormalised Dempster's rule on product spaces and the marginalisation operation whose definitions are provided on [16, p.8] and [16, p.9], respectively.

Proof. From Theorem 1 of [16], using deletion sequence $\mathcal{Z}, \mathcal{H}$, we obtain

$$
\left(m_{\mathfrak{S}}^{\mathcal{Z}} @ m_{\Gamma}^{\mathcal{H} \times \mathcal{Z} \times \mathcal{X}} @ m^{\mathcal{H}}\right)^{\downarrow \mathcal{X}}=\left(\left(m_{\mathfrak{s}}^{\mathcal{Z}} @ m_{\Gamma}^{\mathcal{H} \times \mathcal{Z} \times \mathcal{X}}\right)^{\downarrow \mathcal{H} \times \mathcal{X}} @ m^{\mathcal{H}}\right)^{\downarrow \mathcal{X}} .
$$

Let $m_{\mathfrak{s} \Gamma}^{\mathcal{H} \times \mathcal{Z} \times \mathcal{X}}:=m_{\mathfrak{s}}^{\mathcal{Z}} @ m_{\Gamma}^{\mathcal{H} \times \mathcal{Z} \times \mathcal{X}}$. Eq. (19) may then be rewritten

$$
\left(m_{\mathfrak{s}}^{\mathcal{Z}} @ m_{\Gamma}^{\mathcal{H} \times \mathcal{Z} \times \mathcal{X}} \bigcirc m^{\mathcal{H}}\right)^{\downarrow \mathcal{X}}=\left(m_{\mathfrak{s} \Gamma}^{\mathcal{H} \times \mathcal{Z} \times \mathcal{X} \downarrow \mathcal{H} \times \mathcal{X}} \bigcirc m^{\mathcal{H}}\right)^{\downarrow \mathcal{X}}
$$

We have

$m_{\mathfrak{s} \Gamma}^{\mathcal{H} \times \mathcal{Z} \times \mathcal{X}}(C)= \begin{cases}m_{\mathfrak{s}}^{\mathcal{Z}}\left(\left\{z_{A}\right\}\right) & \text { if } C=\left(\bigcup_{h \in \mathcal{H}, z_{A} \in \mathcal{Z}}\{h\} \times z_{A} \times \Gamma_{z_{A}}(h)\right) \cap\left(\mathcal{H} \times z_{A} \times \mathcal{X}\right) \\ 0 & \text { otherwise. }\end{cases}$

For all $z_{A} \in \mathcal{Z}$ we have

$$
\left[\left(\bigcup_{h \in \mathcal{H}, z_{A} \in \mathcal{Z}}\{h\} \times z_{A} \times \Gamma_{z_{A}}(h)\right) \cap\left(\mathcal{H} \times z_{A} \times \mathcal{X}\right)\right] \downarrow \mathcal{H} \times \mathcal{X}=\bigcup_{h \in \mathcal{H}}\{h\} \times \Gamma_{z_{A}}(h) .
$$

Hence, $m_{\mathfrak{s} \Gamma}^{\mathcal{H} \times \mathcal{Z} \times \mathcal{X} \downarrow \mathcal{H} \times \mathcal{X}}(B)$ for any $B \subseteq \mathcal{H} \times \mathcal{X}$ can be obtained by summing over all $z_{A} \in \mathcal{Z}$ such that $\bigcup_{h \in \mathcal{H}}\{h\} \times \Gamma_{z_{A}}(h)=B$ : 


$$
\begin{aligned}
m_{\mathfrak{s} \Gamma}^{\mathcal{H} \times \mathcal{Z} \times \mathcal{X} \downarrow \mathcal{H} \times \mathcal{X}}(B) & =\sum_{z_{A} \in \mathcal{Z}: \bigcup_{h \in \mathcal{H}}\{h\} \times \Gamma_{z_{A}}(h)=B} m_{\mathfrak{s}}^{\mathcal{Z}}\left(\left\{z_{A}\right\}\right) \\
& =\sum_{A \subseteq \mathcal{Y}: \bigcup_{h \in \mathcal{H}}\{h\} \times \Gamma_{A}(h)=B} m_{\mathfrak{s}}^{\mathcal{Y}}(A) .
\end{aligned}
$$

Since $\exists h \in \mathcal{H}$ such that $\Gamma_{A_{1}}(h) \neq \Gamma_{A_{2}}(h)$ for any two distinct subsets $A_{1}$ and $A_{2}$ of $\mathcal{Y}$, we obtain

$$
m_{\mathfrak{s} \Gamma}^{\mathcal{H} \times \mathcal{Z} \times \mathcal{X} \downarrow \mathcal{H} \times \mathcal{X}}\left[\bigcup_{h \in \mathcal{H}}\left(\{h\} \times \Gamma_{A}(h)\right)\right]=m_{\mathfrak{s}}^{\mathcal{Y}}(A), \quad \forall A \subseteq \mathcal{Y} .
$$

The lemma follows then from Lemma 1 of [24].

Lemma 2 (BBF). Let $m^{\mathcal{H}_{1: 2}}$ and $m_{i}^{\mathcal{Y}}, i=1,2$, be the mass functions in (18). For $i=1,2$, let $\mathcal{Z}_{i}=2^{\mathcal{Y}}$ and let $z_{A}^{i}$ denote the element of $\mathcal{Z}_{i}$ corresponding to the subset A of $\mathcal{Y}, \forall A \subseteq \mathcal{Y}$. For each $A \subseteq \mathcal{Y}$ and $i=1,2$, let $\Gamma_{z_{A}^{i}}$ be the multi-valued mapping from $\mathcal{H}_{i}$ to $\mathcal{X}$ such that $\Gamma_{z_{A}^{i}}(h)=\Gamma_{A}(h)$ for all $h \in \mathcal{H}_{i}$. Let $m_{i}^{\mathcal{Z}_{i}}$ be the mass function defined by $m_{i}^{\mathcal{Z}_{i}}\left(\left\{z_{A}^{i}\right\}\right)=m_{i}^{\mathcal{Y}}(A)$, for all $A \subseteq \mathcal{Y}$. For $i=1,2$, let $m_{\Gamma i}^{\mathcal{H}_{i} \times \mathcal{Z}_{i} \times \mathcal{X}}$ be the mass function defined by $m_{\Gamma i}^{\mathcal{H}_{i} \times \mathcal{Z}_{i} \times \mathcal{X}}\left[\bigcup_{h \in \mathcal{H}_{i}, z_{A}^{i} \in \mathcal{Z}_{i}}\left(\{h\} \times z_{A}^{i} \times \Gamma_{z_{A}^{i}}(h)\right)\right]=1$.

We have, for all $B \subseteq \mathcal{X}$,

$$
\left(m_{1}^{\mathcal{Z}_{1}} @ m_{\Gamma 1}^{\mathcal{H}_{1} \times \mathcal{Z}_{1} \times \mathcal{X}} @ m_{2}^{\mathcal{Z}_{2}} @ m_{\Gamma 2}^{\mathcal{H}_{2} \times \mathcal{Z}_{2} \times \mathcal{X}} @ m^{\mathcal{H}_{1: 2}}\right)^{\downarrow \mathcal{X}}(B)=m^{\mathcal{X}}(B)
$$

where $m^{\mathcal{X}}$ is the mass function defined by (18).

Proof. From Theorem 1 of [16], using deletion sequence $\mathcal{Z}_{1}, \mathcal{Z}_{2}$, we obtain

$$
\begin{aligned}
& \left(m_{1}^{\mathcal{Z}_{1}} \fallingdotseq m_{\Gamma 1}^{\mathcal{H}_{1} \times \mathcal{Z}_{1} \times \mathcal{X}} \curvearrowleft m_{2}^{\mathcal{Z}_{2}} \curvearrowleft m_{\Gamma 2}^{\mathcal{H}_{2} \times \mathcal{Z}_{2} \times \mathcal{X}} \curvearrowleft m^{\mathcal{H}_{1: 2}}\right)^{\downarrow \mathcal{H}_{1} \times \mathcal{H}_{2} \times \mathcal{X}} \\
& =\left(m_{1}^{\mathcal{Z}_{1}} \bigcirc m_{\Gamma 1}^{\mathcal{H}_{1} \times \mathcal{Z}_{1} \times \mathcal{X}}\right)^{\downarrow \mathcal{H}_{1} \times \mathcal{X}} @\left(m_{2}^{\mathcal{Z}_{2}} \bigcirc m_{\Gamma 2}^{\mathcal{H}_{2} \times \mathcal{Z}_{2} \times \mathcal{X}}\right)^{\downarrow \mathcal{H}_{2} \times \mathcal{X}} \curvearrowleft m^{\mathcal{H}_{1: 2}}
\end{aligned}
$$

For $i=1,2$, let $m_{i \Gamma}^{\mathcal{H}_{i} \times \mathcal{X}}:=\left(m_{i}^{\mathcal{Z}_{i}} \bigcirc m_{\Gamma i}^{\mathcal{H}_{i} \times \mathcal{Z}_{i} \times \mathcal{X}}\right)^{\downarrow \mathcal{H}_{i} \times \mathcal{X}}$. Eq. (20) may then be rewritten

$$
\begin{aligned}
& \left(m_{1}^{\mathcal{Z}_{1}} \bigcirc m_{\Gamma 1}^{\mathcal{H}_{1} \times \mathcal{Z}_{1} \times \mathcal{X}} @ m_{2}^{\mathcal{Z}_{2}} \bigcirc m_{\Gamma 2}^{\mathcal{H}_{2} \times \mathcal{Z}_{2} \times \mathcal{X}} \bigcirc m^{\mathcal{H}_{1: 2}}\right)^{\downarrow \mathcal{H}_{1} \times \mathcal{H}_{2} \times \mathcal{X}} \\
& =m_{1 \Gamma}^{\mathcal{H}_{1} \times \mathcal{X}} \oplus m_{2 \Gamma}^{\mathcal{H}_{2} \times \mathcal{X}} \oplus m^{\mathcal{H}_{1: 2}} .
\end{aligned}
$$

The transitivity of marginalisation [16] yields

$$
\begin{aligned}
& \left(\left(m_{1}^{\mathcal{Z}_{1}} \bigcirc m_{\Gamma 1}^{\mathcal{H}_{1} \times \mathcal{Z}_{1} \times \mathcal{X}} \bigcirc m_{2}^{\mathcal{Z}_{2}} \bigcirc m_{\Gamma 2}^{\mathcal{H}_{2} \times \mathcal{Z}_{2} \times \mathcal{X}} \bigcirc m^{\mathcal{H}_{1: 2}}\right)^{\downarrow \mathcal{H}_{1} \times \mathcal{H}_{2} \times \mathcal{X}}\right)^{\downarrow \mathcal{X}} \\
& =\left(m_{1}^{\mathcal{Z}_{1}} \curvearrowleft m_{\Gamma 1}^{\mathcal{H}_{1} \times \mathcal{Z}_{1} \times \mathcal{X}} \curvearrowleft m_{2}^{\mathcal{Z}_{2}} \oplus m_{\Gamma 2}^{\mathcal{H}_{2} \times \mathcal{Z}_{2} \times \mathcal{X}} \curvearrowleft m^{\mathcal{H}_{1: 2}}\right)^{\downarrow \mathcal{X}},
\end{aligned}
$$


from which we obtain, using (21),

$$
\begin{aligned}
& \left(m_{1}^{\mathcal{Z}_{1}} \bigcirc m_{\Gamma 1}^{\mathcal{H}_{1} \times \mathcal{Z}_{1} \times \mathcal{X}} \bigcirc m_{2}^{\mathcal{Z}_{2}} \bigcirc m_{\Gamma 2}^{\mathcal{H}_{2} \times \mathcal{Z}_{2} \times \mathcal{X}} \bigcirc m^{\mathcal{H}_{1: 2}}\right)^{\downarrow \mathcal{X}} \\
& =\left(m_{1 \Gamma}^{\mathcal{H}_{1} \times \mathcal{X}} \bigcirc m_{2 \Gamma}^{\mathcal{H}_{2} \times \mathcal{X}} \bigcirc m^{\mathcal{H}_{1: 2}}\right)^{\downarrow \mathcal{X}} .
\end{aligned}
$$

From the proof of Lemma 1, we have for $i=1,2$,

$$
m_{i \Gamma}^{\mathcal{H}_{i} \times \mathcal{X}}\left[\bigcup_{h \in \mathcal{H}_{i}}\left(\{h\} \times \Gamma_{A}(h)\right)\right]=m_{i}^{\mathcal{Y}}(A), \quad \forall A \subseteq \mathcal{Y}
$$

The lemma follows then from Lemma 2 of [24].

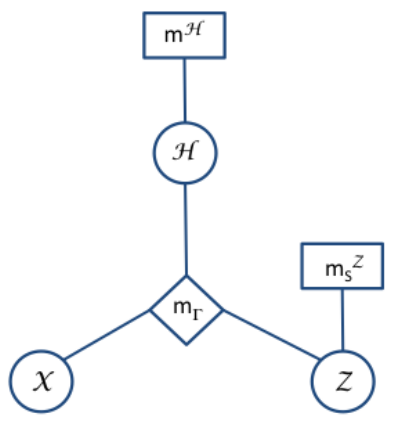

(a)

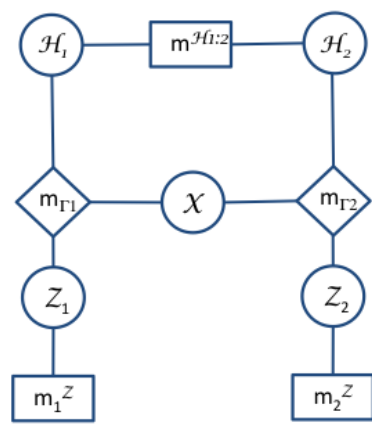

(b)

Fig. 1 Valuation networks corresponding to the BBC procedure (a) and the BBF rule (b).

Lemmas 1 and 2 are instrumental to show Theorem 1, which concerns metaindependent sources.

Theorem 1. With meta-independent sources, it is equivalent to combine the uncertain information $m_{1}^{\mathcal{Y}}$ and $m_{2}^{\mathcal{Y}}$ by the BBF rule or to combine by the conjunctive rule each of these pieces of information corrected using the BBC procedure.

Proof. Let $m^{\mathcal{H}_{1}}$ and $m^{\mathcal{H}_{2}}$ represent meta-knowledge on the two sources $\mathfrak{s}_{1}$ and $\mathfrak{s}_{2}$, respectively. Meta-independence of $\mathfrak{s}_{1}$ and $\mathfrak{s}_{2}$ is equivalent to $m^{\mathcal{H}_{1: 2}}=m^{\mathcal{H}_{1}} \bigcirc m^{\mathcal{H}_{2}}$. Under this assumption, we have, with the same notations as above:

$$
\begin{aligned}
& m_{1}^{\mathcal{Z}_{1}} \bigcirc m_{\Gamma 1}^{\mathcal{H}_{1} \times \mathcal{Z}_{1} \times \mathcal{X}} \bigcirc m_{2}^{\mathcal{Z}_{2}} \bigcirc m_{\Gamma 2}^{\mathcal{H}_{2} \times \mathcal{Z}_{2} \times \mathcal{X}} \bigcirc m^{\mathcal{H}_{1: 2}} \\
& =m_{1}^{\mathcal{Z}_{1}} \oplus m_{\Gamma 1}^{\mathcal{H}_{1} \times \mathcal{Z}_{1} \times \mathcal{X}} \oplus m_{2}^{\mathcal{Z}_{2}} \oplus m_{\Gamma 2}^{\mathcal{H}_{2} \times \mathcal{Z}_{2} \times \mathcal{X}} \curvearrowleft m^{\mathcal{H}_{1}} \oplus m^{\mathcal{H}_{2}} .
\end{aligned}
$$

From Theorem 1 of [16], using deletion sequence $\mathcal{Z}_{1}, \mathcal{Z}_{2}, \mathcal{H}_{2}, \mathcal{H}_{1}$, we obtain 


$$
\begin{aligned}
& \left(m_{1}^{\mathcal{Z}_{1}} @ m_{\Gamma 1}^{\mathcal{H}_{1} \times \mathcal{Z}_{1} \times \mathcal{X}} @ m_{2}^{\mathcal{Z}_{2}} @ m_{\Gamma 2}^{\mathcal{H}_{2} \times \mathcal{Z}_{2} \times \mathcal{X}} @ m^{\mathcal{H}_{1}} @ m^{\mathcal{H}_{2}}\right)^{\downarrow \mathcal{X}} \\
& =\left(\left(m_{1}^{\mathcal{Z}_{1}} @ m_{\Gamma 1}^{\mathcal{H}_{1} \times \mathcal{Z}_{1} \times \mathcal{X}}\right)^{\downarrow \mathcal{H} 1 \times \mathcal{X}} @ m^{\mathcal{H}_{1}}\right)^{\downarrow \mathcal{X}} @\left(\left(m_{2}^{\mathcal{Z}_{2}} @ m_{\Gamma 2}^{\mathcal{H}_{2} \times \mathcal{Z}_{2} \times \mathcal{X}}\right)^{\downarrow \mathcal{H}_{2} \times \mathcal{X}} @ m^{\mathcal{H}_{2}}\right)^{\downarrow \mathcal{X}},
\end{aligned}
$$

which, using (19), can be rewritten

$$
\begin{aligned}
& \left(m_{1}^{\mathcal{Z}_{1}} @ m_{\Gamma 1}^{\mathcal{H}_{1} \times \mathcal{Z}_{1} \times \mathcal{X}} @ m_{2}^{\mathcal{Z}_{2}} @ m_{\Gamma 2}^{\mathcal{H}_{2} \times \mathcal{Z}_{2} \times \mathcal{X}} @ m^{\mathcal{H}_{1}} @ m^{\mathcal{H}_{2}}\right)^{\downarrow \mathcal{X}} \\
& =\left(m_{1}^{\mathcal{Z}_{1}} @ m_{\Gamma 1}^{\mathcal{H}_{1} \times \mathcal{Z}_{1} \times \mathcal{X}} @ m^{\mathcal{H}_{1}}\right)^{\downarrow \mathcal{X}} @\left(m_{2}^{\mathcal{Z}_{2}} @ m_{\Gamma 2}^{\mathcal{H}_{2} \times \mathcal{Z}_{2} \times \mathcal{X}} @ m^{\mathcal{H}_{2}}\right)^{\downarrow \mathcal{X}} .
\end{aligned}
$$

The theorem follows then from Lemmas 1 and 2.

\section{Related works}

The framework described in the previous sections for the correction and fusion of pieces of information is theoretical. It does not include any practical means to apply it and in particular means to obtain the meta-knowledge that it requires. This latter issue is discussed in Section 4.1. In addition, other approaches have been proposed to exploit meta-knowledge about sources. They are related to ours in Section 4.2.

\subsection{Obtaining meta-knowledge}

Both the BBC procedure (17) and the BBF rule (18) require meta-knowledge on the sources, in the form of a mass function $m^{\mathcal{H}}$ on some space $\mathcal{H}$ of assumptions about the sources, where the transformations of a source testimony associated to these assumptions are encoded by multi-valued mappings from $\mathcal{H}$ to $\mathcal{X}$. A central issue is thus to obtain such meta-knowledge. Two main situations can be distinguished with respect to this problem.

First, one may have some prior information about the sources. This information may take the form of data. In particular, one may have access to a confusion matrix counting the correct (crisp and precise) outputs of a source and its errors. As detailed in $[8,17]$, it is possible to estimate the relevance or the truthfulness of a source from such data. If one has access to the uncertain outputs of sources for some known objects, then one may search for the meta-knowledge that induces the least errors [9], and specifically, as shown recently in [26], the meta-knowledge associated with the contextual discounting, contextual reinforcement and contextual negating operations can be learnt efficiently. Prior information about the sources may also take the form of expert knowledge. For instance, schemes using such knowledge and relying on multicriteria aggregations, have been proposed to evaluate the reliability of information sources $[4,25]$. 
In the absence of prior information about the sources, a piece of meta-knowledge that induces a good tradeoff between specificity and consistency of the inferred knowledge about $x$ can be selected [23]. To make this principle operational, it is proposed in [23] to consider an ordered collection $\mathbf{m}^{\mathcal{H}}=\left(m_{1}^{\mathcal{H}}, \ldots, m_{M}^{\mathcal{H}}\right)$ such that the piece of meta-knowledge $m_{1}^{\mathcal{H}}$ corresponds to the conjunctive rule and, for any $1 \leq j<M, m_{j}^{\mathcal{H}}$ induces a more specific knowledge about $x$ than $m_{j+1}^{\mathcal{H}}$ whatever the source testimonies may be. Then, using the fact that $m_{j}^{\mathcal{H}}$ necessarily induces a less consistent knowledge on $x$ than $m_{j+1}^{\mathcal{H}}$, one should test iteratively each $m_{j}^{\mathcal{H}}$ with $j=1, \ldots, M$, and select the first one for which a sufficient degree of consistency is obtained. As illustrated in [23], collection $\mathbf{m}^{\mathcal{H}}$ can be based on important fusion schemes, such as discount and combine, the $r$-out-of- $n$ relevant sources assumption, or the $\alpha$-conjunctions. Besides, this general approach subsumes some classical fusion strategies, and in particular sequential discounting approaches [23].

\subsection{Other modelling approaches}

We have already seen that the approach presented in Section 2.1 extends the discounting operation, which corresponds to the case where the source is known to be truthful, but has only a probability of being relevant. Smets [33] proposed a counterpart to this operation, in which the source is relevant but is not truthful, which is also clearly extended by our approach.

The approach described in Section 3.1, i.e., the BBC procedure, subsumes the ballooning extension and contextual correction mechanisms, as already mentioned. It is also more general than the partially relevant information sources model proposed by Haenni and Hartmann [11], as explained in [24].

An extension of the discounting operation was proposed in [18], in which uncertain meta-knowledge on a source $\mathfrak{s}$ is quantified by a mass function $m^{\mathcal{H}}$ on a space $\mathcal{H}=\left\{h_{1}, \ldots, h_{N}\right\}$ of possible states of the source, as is the case for the BBC procedure. The interpretation of states $h \in \mathcal{H}$ is given in this extension by transforms $m_{h}^{\mathcal{X}}$ of $m_{\mathfrak{S}}^{\mathcal{X}}$ : if the source supplies the uncertain testimony $m_{\mathfrak{s}}^{\mathcal{X}}$ and is in state $h$, then our knowledge on $x$ is represented by $m_{h}^{\mathcal{X}}$. As detailed in [24], this extension and BBC coincide in the special case where the mass function $m^{\mathcal{H}}$ on $\mathcal{H}$ is Bayesian, and $m_{h}^{\mathcal{X}}$ is defined from $m_{\mathfrak{s}}^{\mathcal{X}}$ using multi-valued mappings $\Gamma_{A}$ as: $m_{h}^{\mathcal{X}}(B)=\sum_{A: \Gamma_{A}(h)=B} m_{\mathfrak{s}}^{\mathcal{Y}}(A)$, for all $B \subseteq \mathcal{X}$. Nonetheless, the two models are not equivalent in general, and one should use one model or the other depending on the nature of available knowledge.

Finally, we may remark that alternatives to Dempster's rule, where intersection is replaced with other set operations, have already been considered in [7]. However, the approach of Section 2.2 is the first to provide an explicit interpretation for the resulting rules. In addition, the idea in Section 3.3 of using valuation networks to recover the $\mathrm{BBC}$ procedure and the $\mathrm{BBF}$ rule is inspired from similar approaches $[29,10]$ used to recover the discounting operation and the disjunctive rule. 


\section{Conclusions}

In this chapter, a general approach to the correction and fusion of belief functions has been proposed. It integrates meta-knowledge about the sources of information, that is, knowledge about their quality. An important particular case where metaknowledge concerns the relevance and the truthfulness of the sources has been deeply studied. It significantly extends Shafer's discounting operation and the unnormalized Dempster's rule, which deal only with source relevance. Various forms of lack of truthfulness have also been considered. Specifically, different definitions of the contextual non truthfulness of a source have been introduced. With these definitions, contextual discounting and Smets' $\alpha$-junctions can be seen as special cases of the proposed correction and fusion procedures, respectively. In addition, we have proved that these procedures can be recovered by propagating uncertainty in some particular valuation networks. This result allowed us to show that, when the behaviours of the sources are independent, it is equivalent to combine the sources' information using our fusion procedure or to combine the pieces of information modified using our correction procedure by the unnormalized Dempster's rule. Finally, practical means to apply this general approach have been reviewed, making it a potentially useful tool for various problems, such as combining classifiers or handling intelligence reports.

\section{References}

1. A. P. Dempster. Upper and lower probabilities induced by a multivalued mapping. Annals of Mathematical Statistics, 38:325-339, 1967.

2. T. Denœux. A $k$-nearest neighbor classification rule based on Dempster-Shafer theory. IEEE Transactions on Systems, Man and Cybernetics, 25(5):804-213, 1995.

3. T. Denœux and P. Smets. Classification using belief functions: Relationship between casebased and model-based approaches. IEEE Transactions on Systems, Man, and Cybernetics, Part B, 36(6): 1395-1406, 2006.

4. S. Destercke, P. Buche, and B. Charnomordic. Evaluating data reliability : an evidential answer with application to a web-enabled data warehouse. IEEE Transactions on Knowledge and Data Engineering, 25(1):92-105, 2013.

5. S. Destercke and D. Dubois. Idempotent conjunctive combination of belief functions: Extending the minimum rule of possibility theory. Information Sciences, 181(18):3925 - 3945, 2011.

6. D. Diderot and J. le Rond d'Alembert, editors. Encyclopédie, ou dictionnaire raisonné des sciences, des arts et des métiers. University of Chicago: ARTFL Encyclopédie Project (Spring 2016 Edition), R. Morrissey and G. Roe (eds), http://encyclopedie.uchicago.edu/.

7. D. Dubois and H. Prade. A set-theoretic view of belief functions: logical operations and approximations by fuzzy sets. International Journal of General Systems, 12(3):193-226, 1986.

8. Z. Elouedi, E. Lefèvre, and D. Mercier. Discountings of a belief function using a confusion matrix. In 22nd IEEE International Conference on Tools with Artificial Intelligence (ICTAI), volume 1, pages 287-294, Oct 2010.

9. Z. Elouedi, K. Mellouli, and P. Smets. Assessing Sensor Reliability for Multisensor Data Fusion Within the Transferable Belief Model. IEEE Transactions on Systems, Man and Cybernetics, Part B, 34(1):782-787, 2004. 
10. R. Haenni. Uncover Dempster's rule where it is hidden. In Proceedings of the 9th International Conference on Information Fusion (FUSION 2006), Florence, Italy, 2006.

11. R. Haenni and S. Hartmann. Modeling partially reliable information sources: a general approach based on Dempster-Shafer theory. Information Fusion, 7(4):361-379, 2006.

12. R. D. Huddleston and G. K. Pullum. A Student's Introduction to English Grammar. Cambridge University Press, 2005.

13. F. Janez and A. Appriou. Théorie de l'évidence et cadres de discernement non exhaustifs. Traitement du Signal, 13(3):237-250, 1996.

14. F. Janez and A. Appriou. Theory of evidence and non-exhaustive frames of discernment : Plausibilities correction methods. International Journal of Approximate Reasoning, 18:1-19, 1998.

15. J. Klein and O. Colot. Automatic discounting rate computation using a dissent criterion. In Proceedings of the 1st Workshop on the Theory of Belief Functions (BELIEF), pages 1-6, 2010.

16. J. Kohlas and P. P. Shenoy. Computation in valuation algebras. In D. M. Gabbay and Ph. Smets, editors, Handbook of Defeasible Reasoning and Uncertainty Management Systems: Algorithms for Uncertainty and Defeasible Reasoning, volume 5, pages 5-39. Kluwer, Dordrecht, 2000.

17. E. Lefèvre, F. Pichon, D. Mercier, Z. Elouedi, and B. Quost. Estimation de sincérité et pertinence à partir de matrices de confusion pour la correction de fonctions de croyance. In Rencontres Francophones sur la Logique Floue et ses Applications (LFA), volume 1, pages 287-294. Cépaduès, Nov 2014.

18. D. Mercier, T. Denœux, and M.-H. Masson. Belief function correction mechanisms. In B. Bouchon-Meunier, R. R. Yager, J.-L. Verdegay, M. Ojeda-Aciego, and L. Magdalena, editors, Foundations of Reasoning under Uncertainty, volume 249 of Studies in Fuzziness and Soft Computing, pages 203-222. Springer-Verlag, Berlin, 2010.

19. D. Mercier, E. Lefèvre, and F. Delmotte. Belief functions contextual discounting and canonical decompositions. International Journal of Approximate Reasoning, 53(2):146-158, 2012.

20. D. Mercier, F. Pichon, and E. Lefèvre. Corrigendum to "Belief functions contextual discounting and canonical decompositions" [International Journal of Approximate Reasoning 53 (2012) 146-158]. International Journal of Approximate Reasoning, 70:137-139, 2016.

21. D. Mercier, B. Quost, and T. Denœux. Refined modeling of sensor reliability in the belief function framework using contextual discounting. Information Fusion, 9(2):246 - 258, 2008.

22. F. Pichon. On the $\alpha$-conjunctions for combining belief functions. In T. Denœux and M.H. Masson, editors, Belief Functions: Theory and Applications, volume 164 of Advances in Intelligent and Soft Computing, pages 285-292. Springer Berlin / Heidelberg, 2012.

23. F. Pichon, S. Destercke, and T. Burger. A consistency-specificity trade-off to select source behavior in information fusion. IEEE Transactions on Cybernetics, 45(4):598-609, 2015.

24. F. Pichon, D. Dubois, and T. Denœux. Relevance and truthfulness in information correction and fusion. International Journal of Approximate Reasoning, 53(2):159-175, 2012.

25. F. Pichon, C. Labreuche, B. Duqueroie, and T. Delavallade. Multidimensional approach to reliability evaluation of information sources. In P. Capet and T. Delavallade, editors, Information Evaluation, pages 129-156. John Wiley \& Sons, Inc., Hoboken, NJ, USA, 2014.

26. F. Pichon, D. Mercier, E. Lefèvre, and F. Delmotte. Proposition and learning of some belief function contextual correction mechanisms. International Journal of Approximate Reasoning, 72:4-42, 2016.

27. J. Schubert. Conflict management in Dempster-Shafer theory using the degree of falsity. International Journal of Approximate Reasoning, 52(3):449-460, 2011.

28. G. Shafer. A mathematical theory of evidence. Princeton University Press, Princeton, N.J., 1976.

29. P. Smets. Belief functions: the disjunctive rule of combination and the generalized Bayesian theorem. International Journal of Approximate Reasoning, 9(1):1-35, 1993.

30. P. Smets. The $\alpha$-junctions: combination operators applicable to belief functions. In D. M. Gabbay, R. Kruse, A. Nonnengart, and H. J. Ohlbach, editors, First International Joint Confer- 
ence on Qualitative and Quantitative Practical Reasoning (ECSQARU-FAPR'97), Bad Honnef (Germany), 09/06/1997-12/06/1997, volume 1244 of Lecture Notes in Computer Science, pages 131-153. Springer, 1997.

31. P. Smets. The transferable belief model for quantified belief representation. In D. M. Gabbay and $\mathrm{Ph}$. Smets, editors, Handbook of Defeasible Reasoning and Uncertainty Management Systems, volume 1, pages 267-301. Kluwer, Dordrecht, 1998.

32. P. Smets and R. Kennes. The Transferable Belief Model. Artificial Intelligence, 66:191-243, 1994.

33. Ph. Smets. Managing deceitful reports with the transferable belief model. In 8th International Conference on Information Fusion, Philadelphia, USA, July 2005. doi: 10.1109/ICIF.2005.1591953.

34. Y. Yang, D. Han, and C. Han. Discounted combination of unreliable evidence using degree of disagreement. International Journal of Approximate Reasoning, 54(8):1197-1216, 2013. 